Introduction/Background* Epithelial ovarian cancer (EOC) patients undergoing splenectomy during cytoreductive surgery represent a small subgroup of patients. Splenic metastases or technical reasons due to extensive upper abdominal disease may require a splenectomy. However, there is limited knowledge on the impact of splenectomy on the long-term outcome of patients. Particularly, it has been hypothesized that as the antitumor immunologic functions of the spleen may inhibit cancer growth, splenectomy may promote the growth of residual disease during the postoperative period as observed in murine models of other cancer types [3-6]. The aim of this study was to assess the effect of a splenectomy during cytoreductive surgery on perioperative outcomes and survival of advanced stage EOC patients.

Methodology In this nationwide population-based study, all consecutive patients diagnosed with FIGO stage IIIC and IV EOC between 01-01-2008 and 31-12-2015 were identified from the Netherlands Cancer Registry. Patients who underwent cytoreductive surgery combined with platinum-based chemotherapy as primary treatment were selected. Differences in clinicopathological characteristics between splenectomy and non-splenectomy patients were assessed. Progression-free survival (PFS) and overall survival (OS) were analyzed using Kaplan-Meier survival curves and log-rank tests. Cox proportional hazards models were used to adjust for covariates that influence survival.

Result(s)* A total of 3,911 patients were identified, including 99 splenectomy and 3,812 non-splenectomy patients. Splenectomy patients were more likely to undergo extensive surgery or surgical reintervention, to receive intraperitoneal chemotherapy, intraoperative and postoperative blood transfusion, to experience postoperative infections, and to be admitted to Intensive Care Unit (all $\mathrm{p}<0.002)$. No significant differences in PFS and OS were observed between splenectomy versus non-splenectomy patients after adjusting for covariates.

*An additional 163 patients were excluded from the survival analysis with reference to figure 1 , since these patients had unknown follow-up or survival data.

*An additional 10 patients were excluded from the survival analysis with reference to figure 1 , since these patients had unknown follow-up or survival data.

Conclusion* While advanced stage EOC patients who undergo splenectomy during cytoreductive surgery have more extensive

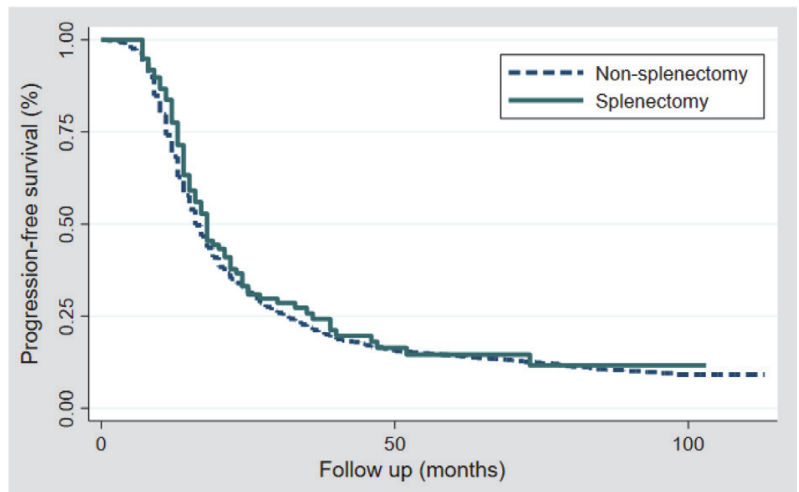

Abstract 770 Figure 1 Kaplan-Meier estimates of the progressionfree survival (PFS) of non-splenectomy patients ( $N=3,654$, dash line) and splenectomy patients ( $N=94$, solid line). The median PFS in months were 16 and 18 months for the non-splenectomy and splenectomy patients, respectively. No significant difference in PFS was observed with the log-rank test $(p=0.477)$.

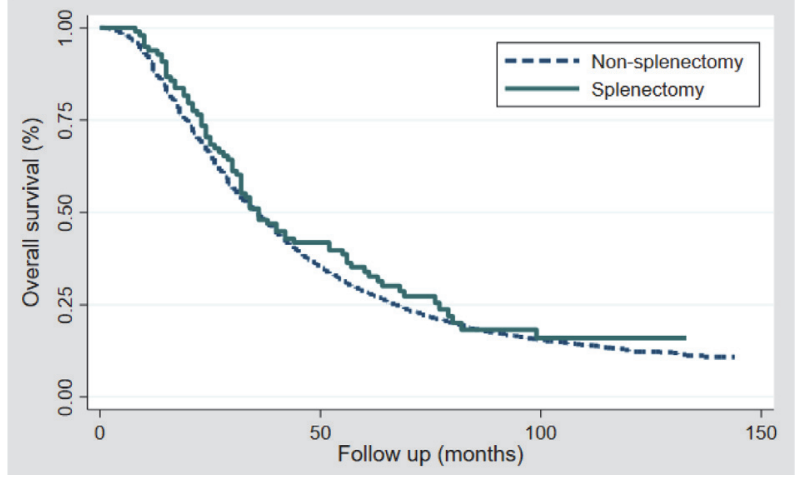

Abstract 770 Figure 2 Kaplan-Meier estimates of the overall survival (OS) of non-splenectomy patients ( $N=3,803$, dash line) and splenectomy patients ( $\mathrm{N}=98$, solid line). The median OS in months was 36 months for both the non-splenectomy and splenectomy patients. No significant difference in OS was observed with the log-rank test $(p=0.306)$.

disease and undergo intensive treatment, no adverse effect of splenectomy on the survival was observed. Splenectomy during cytoreductive surgery is justified to achieve complete cytoreduction in advanced stage EOC patients.

\section{CHANGES IN THEATRE UTILISATION FOLLOWING IMPLEMENTATION AND ESTABLISHMENT OF MAXIMAL EFFORT SURGICAL PHILOSOPHY FOR ADVANCED EPITHELIAL OVARIAN CANCER}

I Mantrali* , A Phillips, S Abdul, V Asher, A Bali. Royal Derby Hospital, UK

\subsection{6/ijgc-2021-ESG0.468}

Introduction/Background* The most significant prognostic factors in AOC are platinum sensitivity and residual disease following cytoreductive surgery. In order to achieve complete (R0) cytoreduction advanced surgical procedures may be required, so called ultraradical (UR) surgery. Such a change from traditional pelvic centred surgery to abdominal and thoracic wide surgery increases operative time. We aimed to evaluate the change in operative time (OT) following the establishment of a maximum effort treatment paradigm.

Methodology We performed a retrospective study looking at all stage 3/4 epithelial ovarian, tubal and peritoneal cancers operated on between 2014 and 2020 inclusive in one Cancer Centre in the UK. 198 patients were looked at with two patients excluded for missing variables. Surgical complexity was classified by the surgical complexity score as advocated by Aletti. OT was measured in minutes. Continuous variables were expressed as mean and standard deviation. Categorical variables were analysed using chi-squared testing. Groups were compared using one-way ANOVA.

Result(s)* A total of 196 were included. Results identified increasing year-on-year OT (2014; $192 \pm 88$ vs. 2020; 426 $\pm 121, \mathrm{p}<0.0001)$. In latter years, OT plateaued $(2018 ; 366$ \pm 131 vs. $2020 ; 426 \pm 121, \mathrm{p}>0.05)$. During this time period the R0 rate increased from $61 \%$ to $96 \%, \mathrm{p}=0.001$, achieved by more UR procedures, which increased from $13 \%$ to $71 \%, \mathrm{p}<0.0001$ and intermediate/high complexity surgery from $34 \%$ to $86 \%, p=0.0003$. In 2014 , when more standard procedures were being performed, mean OT was approximately 3 hours, excluding anaesthetic time (2014; mean OT 
$192+/-88)$. As UR Surgeries increased to $>50 \%$ of overall number of surgeries for advanced disease, the mean OT was a full operating day $(2018 ; 367 \pm 132,2019 ; 375 \pm 153$ and $2020 ; 426 \pm 122)$. Further increases in UR rates had less impact on OT $(\mathrm{F}(3,62)=1.11, \mathrm{p}>0.05)$.

Conclusion* Effectively treating AOC patients requires appropriate theatre resources. We recommend that the standard of care for cytoreductive surgeries operated with maximal effort should be that only case is performed on that day. Funding and resource allocation for ovarian cancer surgery in the United Kingdom needs to reflect the greater theatre utilisation needed for these patients.

\section{ROLE OF OPTIMAL CYTOREDUCTION IN ADVANCED STAGE MALIGNANT OVARIAN GERM CELL TUMORS, DATA FROM THE MITO-9 TRIAL}

${ }^{1} \mathrm{~A}$ Bergamini*, ${ }^{2} \mathrm{C}$ Cassani, ${ }^{3} \mathrm{~F}$ Raspagliesi, ${ }^{3} \mathrm{G}$ Bogani, ${ }^{1} \mathrm{~L}$ Bocciolone, ${ }^{1} \mathrm{E}$ Rabaiotti, ${ }^{1} \mathrm{R}$ Cioffi, ${ }^{1} \mathrm{G}$ Sabetta, ${ }^{4} \mathrm{G}$ Scarfone, ${ }^{5} \mathrm{~A}$ Savarese, ${ }^{6} \mathrm{MG}$ Ferrandina, ${ }^{6} \mathrm{~F}$ Mascilini, ${ }^{7} \mathrm{G}$ Cormio, ${ }^{8,9} \mathrm{G}$ Giorda, ${ }^{10} \mathrm{~S}$ Pignata, ${ }^{1} \mathrm{G}$ Taccagni, ${ }^{1} \mathrm{G}$ Mangili. ${ }^{1}$ IRCCS San Raffaele Hospital, Obstetrics and Gynecology, Milan; ${ }^{2}$ Fondazione I. R.C.C.S. Policlinico San Matteo, Pavia, Italy; ${ }^{3}$ stituto Nazionale dei Tumori | Fondazione IRCCS, Milano, Italy; ${ }^{4}$ Mangiagalli Clinic IRCCS Cà Granda Foundation Ospedale Maggiore Policlinico, Milano, Italy; ${ }^{5}$ Ospedale Regina Elena, Roma, Italy; ${ }^{6}$ Agostino Gemelli University Policlinic, Roma, Italy; ${ }^{7}$ Ospedale Oncologico Giovanni Paolo II, Bari, Italy; ${ }^{8}$ Aviano, Italy; ${ }^{9}$ Oncology Referral Center, Aviano, Italy; ${ }^{10}$ IRCCS Istituto Nazionale Tumori "Fondazione G. Pascale"

10.1136/ijgc-2021-ESGO.469

Introduction/Background" the role of optimal cytoreduction in advanced stage MOGCTs is still a debated issue, considering the high chemosensitivity of these tumors and young patients' age. The aim of the present study was to analyze the role of residual tumor at primary surgery in advanced stage MOGCTs.

Methodology clinicopathological data from patients with stage III-IV MOGCTs were retrospectively collected among MITO centers (Multicenter Italian Trials in Ovarian cancer and gynecologic malignancies) and analyzed. All patients underwent primary surgery. Optimal cytoreduction was defined as macroscopic residual tumor $=0$. All patients received adjuvant platinum-based chemotherapy. Clinicopathological features were evaluated for association with relapse.

Result(s)* 42 patients were included. 18 (42.8\%) were affected by dysgerminomas, $24(57.2 \%)$ by non-disgerminomatous MOGCTs. 24 patients (57.2\%) achieved optimal cytoreduction at primary surgery (residual tumor $=0$ ). No statistical difference was detected in stage distribution, age, rate of optimal cytoreduction between the dysgerminomas and non dysgerminomatous groups. Median 5year- disease free survival (DFS) rates were $87.8 \%$ and $50.0 \%$ for dysgerminomas and non-dysgerminomas, respectively. In the whole cohort, there was no difference in terms of 5 years-DFS according to residual tumor at primary surgery $(74.6 \%$ vs $56.0 \%, \mathrm{p}=0.25)$. Residual tumor at primary surgery was a prognostic factor for DFS only for non-dysgerminomatous MOGCTs (64\% vs 30\% for optimal vs suboptimal cytoreduction, respectively, $\mathrm{p}=0.05)$.

Conclusion* in the present study, optimal cytoreduction at primary surgery was a prognostic factor affecting disease-free survival only for non-disgerminomatous MOGCTs.

\section{IS THE SEROUS TUBAL INTRAEPITHELIAL CARCINOMA TO BLAME FOR PERITONEAL CARCINOMATOSIS AFTER RISK-REDUCING SALPINGO-OOPHORECTOMY?}

${ }^{1} M P$ Steenbeek*, ${ }^{2} \mathrm{~J}$ Bulten, 'S Study Group, ${ }^{3}$ RPMG Hermens, ${ }^{3} \mathrm{I}$ Inthout, ${ }^{1} \mathrm{JA}$ De Hullu. ${ }^{1}$ Radboud university medical center, Obstetrics and Gynaecology, Nijmegen, Netherlands; ${ }^{2}$ Radboud university medical center, Pathology, Nijmegen, Netherlands; ${ }^{3}$ Radboud university medical center, Scientific Institute for Quality of Healthcare, Nijmegen, Netherlands

\subsection{6/ijgc-2021-ESG0.470}

Introduction/Background* After risk-reducing salpingo-oophorectomy (RRSO), there is an approximate 3.9\% and $1.9 \%$ risk of developing peritoneal carcinomatosis (PC) for BRCA1/2

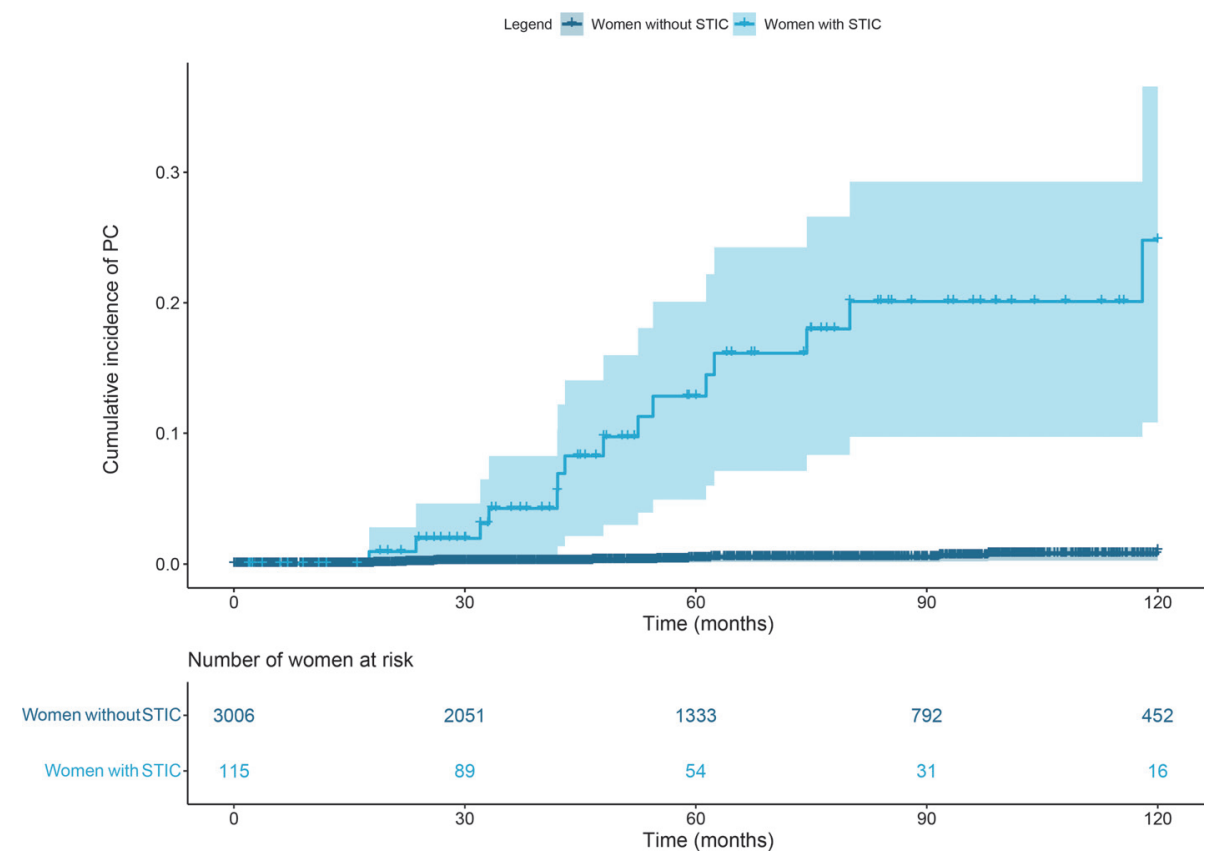

Abstract 781 Figure 1 The incidence of PC in women with and without STIC at RRSO. 